\title{
Low-Dose Paclitaxel Inhibits Tumor Cell Growth by Regulating Glutaminolysis in Colorectal Carcinoma Cells
}

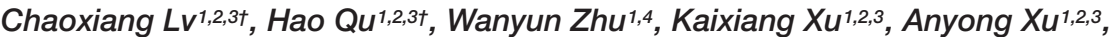 \\ Baoyu Jia ${ }^{1}$, Yubo Qing ${ }^{1}$, Honghui Li ${ }^{1}$, Hong-Jiang Wei ${ }^{1 *}$ and Hong-Ye Zhao ${ }^{1,2,3,4 *}$
}

${ }^{1}$ State Key Laboratory for Conservation and Utilization of Bio-Resources in Yunnan, Yunnan Agricultural University, Kunming, China, ${ }^{2}$ Key Laboratory of Agricultural Biodiversity and Plant Disease Management of China Education Ministry, Yunnan Agricultural University, Kunming, China, ${ }^{3}$ College of Plant Protection, Yunnan Agricultural University, Kunming, China,

${ }^{4}$ College of Pharmacy and Chemistry, Dali University, Dali, China

OPEN ACCESS

Edited by:

Owen McCarty,

Oregon Health \& Science University,

USA

Reviewed by:

Xin Wang,

Stanford University, USA

Ying Luo,

Kunming University of Science and Technology, China

${ }^{*}$ Correspondence: Hong-Ye Zhao hyzhao2000@126.com

Hong-Jiang We

hongjiangwei@126.com

${ }^{\dagger}$ These authors have contributed equally to this work.

Specialty section:

This article was submitted to Cancer Molecular Targets and Therapeutics, a section of the journa Frontiers in Pharmacology

Received: 24 December 2016 Accepted: 18 April 2017 Published: 04 May 2017

Citation:

Lv C, Qu H, Zhu W, Xu K, Xu A,

Jia B, Qing Y, Li H, Wei H-J and Zhao H-Y (2017) Low-Dose Paclitaxel

Inhibits Tumor Cell Growth by Regulating Glutaminolysis in Colorectal Carcinoma Cells.

Front. Pharmacol. 8:244. doi: 10.3389/fphar.2017.00244
Paclitaxel (PTX) is a natural alkaloid isolated from the bark of a tree, Taxus brevifolia, and is currently used to treat a variety of tumors. Recently, it has been found that low-dose PTX is a promising treatment for some cancers, presenting few side effects. However, antitumor mechanisms of low-dose PTX $(<1 \mathrm{nM})$ have rarely been illuminated. Here we report a new antitumor mechanism of low-dose PTX in colorectal carcinoma cells. We treated colorectal carcinoma HCT116 cells with PTX at 0.1 and $0.3 \mathrm{nM}$ for 0,1 , 2 , or 3 days, and found that low-dose PTX inhibits cell growth without altering cell morphology and cell cycle. There was a significant decrease of $\mathrm{pH}$ in culture media with 0.3 nM PTX for 3 days. Also, lactate production was significantly increased in a dose- and time-dependent manner. Furthermore, expression of glutaminolysis-related genes GLS, SLC7A11 and SLC1A5 were significantly decreased in the colorectal carcinoma cells treated with low-dose PTX. Meanwhile, protein expression levels of p53 and p21 increased significantly in colorectal carcinoma cells so treated. In summary, low-dose PTX down-regulated glutaminolysis-related genes and increased their lactate production, resulting in decreased $\mathrm{pH}$ of tumor microenvironments and inhibition of tumor cell growth. Up-regulation of p53 and p21 in colorectal carcinoma cells treated with low-dose PTX also contributed to inhibition of tumor cell growth.

Keywords: low-dose PTX, cell growth, lactate production, glutaminolysis, colorectal carcinoma cells

\section{INTRODUCTION}

Paclitaxel (PTX) has been used for treating various malignancies. It stabilizes polymerized microtubules and enhances microtubule assembly and thus, arrests the cell cycle and induces apoptosis (Barbuti and Chen, 2015). Despite PTX being often indicated as a first-line therapy for treating some cancers, some side effects of high-dose PTX including peripheral neuropathy and anaphylactic hypersensitivity reactions have limited its clinical application (Banerji et al., 2014; Duggett et al., 2016). Recently, low-dose metronomic PTX chemotherapy has attracted increasing attention as an alternative scheme for treatment of some cancers (Lien et al., 2013) because of its high efficiency, low toxicity and reduced drug resistance in clinical settings (Loven et al., 2013; Gong et al., 2014). Anticancer mechanisms of low-dose metronomic PTX include inhibition of 
progress and metastasis of cancer through its strong antiangiogenic and anti-lymphangiogenic activities, rather than by inducing tumor cell apoptosis (Jiang et al., 2010; Bocci et al., 2013). In addition, our previous studies revealed that 1 and $3 \mathrm{nM}$ PTX inhibit the growth of colorectal carcinoma cells by downregulating $\mathrm{c}-\mathrm{Myc}$, and those concentrations did not damage the intestinal crypt epithelial IEC-6 cells ( $\mathrm{Li}$ et al., unpublished). However, antitumor mechanisms of low-dose PTX ( $<1 \mathrm{nM})$ have seldom been reported.

Tumor cell proliferation requires sufficient energy to synthesis macromolecules, and need to be allocated into appropriate metabolic pathways to produce energy and maintain cell growth (Gentric et al., 2016). It has been reported that glucose metabolism and glutamine metabolism together account for most of the carbon and nitrogen metabolism in mammalian cells (Polet et al., 2016). Glycolysis is a major energy metabolism pathway for tumor cells, and targeting this pathway is a potential strategy to inhibit cancer cell growth. The pathway involves multiple genes, for example, the solute carrier family 2 member 1 (GLUT1) gene brings extracellular glucose into the cytoplasm, hexokinase 1 (HK1) genes phosphorylate intracellular glucose to form glucose-6-phosphate. Other genes, such as phosphoglycerate dehydrogenase $(P H G D H)$ and pyruvate dehydrogenase kinase1 (PDK1), also play roles in glycolysis (Chen et al., 2016). Meanwhile, glutaminolysis also provides carbon and nitrogen to support biosynthetic homeostasis that cancer cells may exploit for growth (Altman et al., 2016). In this process, glutaminase (GLS), which converts glutamine to glutamate, plays a key role in cancer cell metabolism, growth, and proliferation (Kung et al., 2011). The glutamate dehydrogenase1 (GDH) encodes a trifunctional protein that is associated with the enzymatic activities of glutaminolysis (Koo and Yoon, 2015). The solute carrier family 1 member 5 (SLC1A5) is related to glutamine uptake (Bolzoni et al., 2016). The solute carrier family 7 member 11 (SLC7A11) is responsible for glutamate release in glutaminolysis (Kalivas, 2009). Therefore, a comprehensive understanding of the regulation of metabolic fluxes in tumor cell proliferation would contribute to the development of new strategies to treat cancer.

Colorectal carcinoma, a very common malignant tumor of the digestive system, represents a major cause of global cancerrelated deaths (Abdifard et al., 2016). Despite several treatment options for colorectal carcinoma, response rates are relatively low and recurrence is high (Zhou et al., 2016). Chemotherapy, which has limited efficacy and poor prognoses, is currently the primary option for treating colorectal cancer (Jagadish et al., 2016). It has been reported that PTX is effective in the treatment of colorectal cancer (Zhang et al., 2016). Recent studies have shown that PTX can affect glucose metabolism in triple negative breast cancer (Stewart et al., 2016), and that PTX redirects metabolic reprogramming from glycolysis to oxidative phosphorylation, leading to effective suppression of ovarian cancer stem cells (Shen et al., 2015).

However, few studies have investigated effects of low-dose PTX $(<1 \mathrm{nM})$ on glycolysis and glutaminolysis in colorectal carcinoma cells. Here we found that low-dose PTX could inhibit cell growth by regulating glutaminolysis as well as p53 and p21 expression in colorectal carcinoma cells. These findings also illustrate some antitumor mechanisms of low-dose PTX action on cancer cells.

\section{MATERIALS AND METHODS}

\section{Reagents and Antibodies}

Paclitaxel was from Sigma-Aldrich Inc. (St. Louis, MO, USA). Antibodies against p21 and p53 were from Abcam (Burlingame, CA, USA). HK1, PHGDH, GLS, SLC7A11, SLC1A5, and GDH were from Absin Bioscience Inc. (Shanghai, China). $\beta$-actin was from Sigma-Aldrich Inc. (St. Louis, MO, USA).

\section{Cell Lines and Cell Cultures}

Human colorectal carcinoma cell line HCT116 was from the Cell Collection of the Chinese Academy of Sciences (Shanghai, China) and cultured in DMEM-F-12 medium (HyClone, Logan, UT, USA) supplemented with heat-inactivated $10 \%$ fetal bovine serum (Gibco, Carlsbad, CA, USA) and $100 \mathrm{IU} / \mathrm{mL}$ penicillin (Solarbio, Beijing, China). The cells were seeded into a gelatincoated $75-\mathrm{cm}^{2}$ flask and cultured in $10 \mathrm{~mL}$ of medium at $37^{\circ} \mathrm{C}$ in a humidified atmosphere of $5 \% \mathrm{CO}_{2}$ in air.

\section{Cell Morphological Observation}

Exponentially growing HCT116 cells were transferred to 12 -well plates and cultured at $37^{\circ} \mathrm{C}$ in a $5 \% \mathrm{CO}_{2}$ atmosphere. Cells were treated with 0.1 and $0.3 \mathrm{nM}$ PTX for 1-3 days. When the cells were at 60 to $70 \%$ confluence, they were rinsed twice with PBS, and the supernatant was discarded. Then, images were taken using an OLYMPUS IX 71 microscope $(10 \times 10)$ (OLYMPUS, Tokyo, Japan).

TABLE 1 | Primer sequences for q-PCR.

\begin{tabular}{|c|c|}
\hline Gene Name & Primer Sequence $\left(5^{\prime}\right.$ to $\left.3^{\prime}\right)$ \\
\hline \multirow[t]{2}{*}{ LDHA } & F: 5'-ATTTCACTGTCTAGGCTACAACA-3' \\
\hline & R: 5'-TTAATACCATCCAGCATCAGG-3' \\
\hline \multirow[t]{2}{*}{ HK1 } & F: 5'-GGAGCCACCACTCACCCTACT-3' \\
\hline & R: 5'-GGAGCCCATTGTCCGTTACTT-3' \\
\hline \multirow[t]{2}{*}{ PHGDH } & F: 5'-ACCCTGCAATGCTGCCTACCA-3' \\
\hline & R: 5'-ACATGCTGCTTCCACGCTTCC-3' \\
\hline \multirow[t]{2}{*}{ PDK1 } & F: 5'-ATCACCAGGACAGCCAATACA-3' \\
\hline & R: 5'-TCCTCGGTCACTCATCTTCAC-3' \\
\hline \multirow[t]{2}{*}{ GLUT1 } & F: 5'-GACATTCAAGGCATTTCTATCACAT-3' \\
\hline & R: 5'-CGACTTCAGGCACATAACCTCTाT-3' \\
\hline \multirow[t]{2}{*}{$\mathrm{GDH}$} & F: 5'-AATGCTGGAGGAGTGACAGTATCTTA-3' \\
\hline & R: 5'-CTTGGAACTCTGCCGTGGGTA-3' \\
\hline \multirow[t]{2}{*}{ GLS } & F: 5'-GAGTACTGAGCCCTGAAGCAGTTCG-3' \\
\hline & R: 5'GGAGACCAGCACATCATACCCA-3' \\
\hline \multirow[t]{2}{*}{ SLC7A11 } & F: 5'-AGAGGGTCACCTTCCAGAAAT-3' \\
\hline & R: 5'-AGATAAATCAGCCCAGCAACT-3' \\
\hline \multirow[t]{2}{*}{ SLC1A5 } & F: 5'-GAGAAATATCTTCCCTTCCAACCTGG-3' \\
\hline & R: 5'-CCAAAGACGATGGCAAACACTACC-3' \\
\hline \multirow[t]{2}{*}{$\beta$-actin } & F: 5'-CTTAGTTGCGTTACACCCTTCTTG-3' \\
\hline & R: 5'-TCACCTTCACCGTTCCAGTIT-3' \\
\hline
\end{tabular}




\section{A Days}

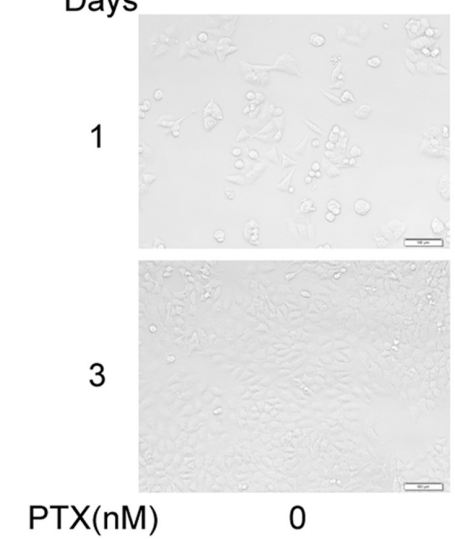

B

$72 \mathrm{~h}$

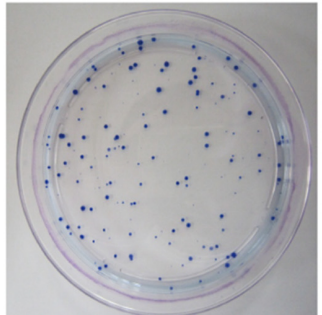

$\operatorname{PTX}(\mathrm{nM})$

0

C

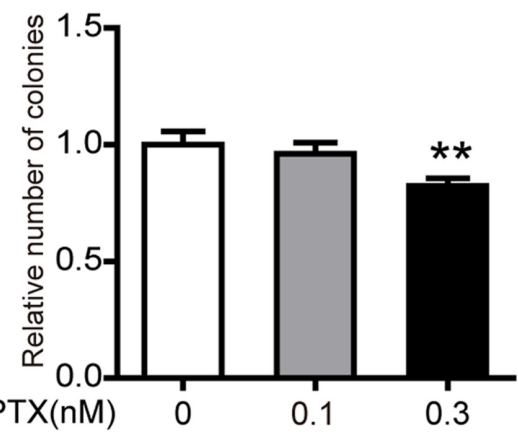

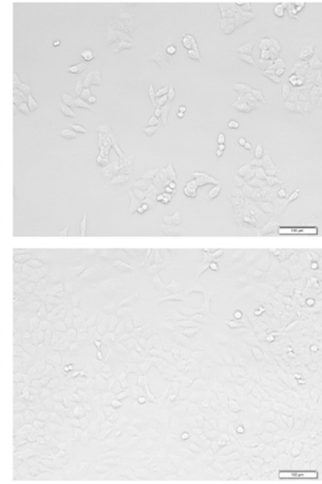

0.1

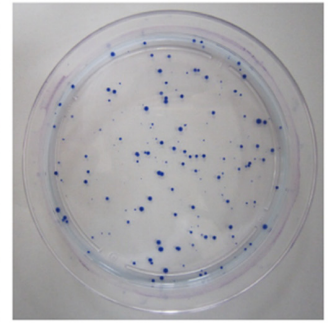

0.1

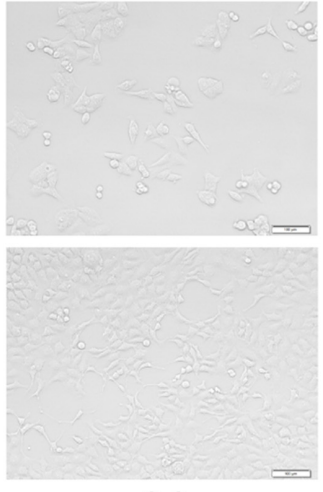

0.3

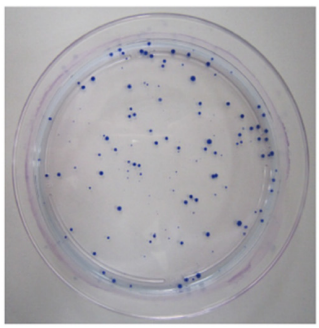

0.3

D

FIGURE 1 | Effect of low-dose PTX on the sensitivity of HCT116 cells. (A) Photomicrographs of HCT116 cells exposed to 0.1 and 0.3 nM PTX for 1 or 3 days (original magnification, $100 \times$ ). (B) Cell viability was detected by colony formation assay. (C) Results of cellular colony formation rate was expressed as a fold change. (D) Quantification of cell viability by MTT assay. Results are shown as mean $\pm \mathrm{SD}$. Date are representative of three independent experiments. (** $p<0.01)$.

\section{Colony Formation Assay}

Cells in the logarithmic growth phase were digested into a single-cell suspension with a trypsin-EDTA (Solarbio, Tongzhou District, Beijing, China) solution, and then $10 \mathrm{~mL}$ of the cell suspension was seeded onto $9 \mathrm{~cm}$ culture plates (NEST, Wuxi, Jiangsu, China) at a density of 20 cells $/ \mathrm{mL}$. After adherence, these cells were treated with PTX $(0.1$ and $0.3 \mathrm{nM})$ for $72 \mathrm{~h}$ and then cultured for 15 days. Thereafter, the cells were fixed in formaldehyde and stained with $10 \%$ Giemsa stain (Solarbio, Tongzhou District, Beijing, China). After multiple washes, the plates were air dried and imaged. Individual clones were counted, and statistical analyses were performed.

\section{Cell Survival by the MTT Assay}

The 3- (4,5-dimethylthiazol-2-yl)-2,5- diphenyltetrazolium bromide (MTT) colorimetric assay was used to determine cytotoxicity of PTX. HCT116 cells were plated at a density of 1000 and 2000 cells/well in 96-well plates and were incubated either with or without PTX at 0.1 or $0.3 \mathrm{nM}$ for 3 days. Then, $50 \mu \mathrm{L}$ of MTT $1 \mathrm{mg} / \mathrm{mL}$ (Sigma-Aldrich) tetrazolium substrate was added to each well, and plates were incubated for an additional $4 \mathrm{~h}$ at $37^{\circ} \mathrm{C}$. The resulting violet formazan precipitate was solubilized by adding $100 \mu \mathrm{L}$ of $50 \% \mathrm{~N}, \mathrm{~N}-$ dimethylformamide. All of these plates were shaken for $5 \mathrm{~min}$ and read immediately at $578 \mathrm{~nm}$ using a model 550 Micro Plate Reader (Bio-Rad, Richmond, CA, USA). 

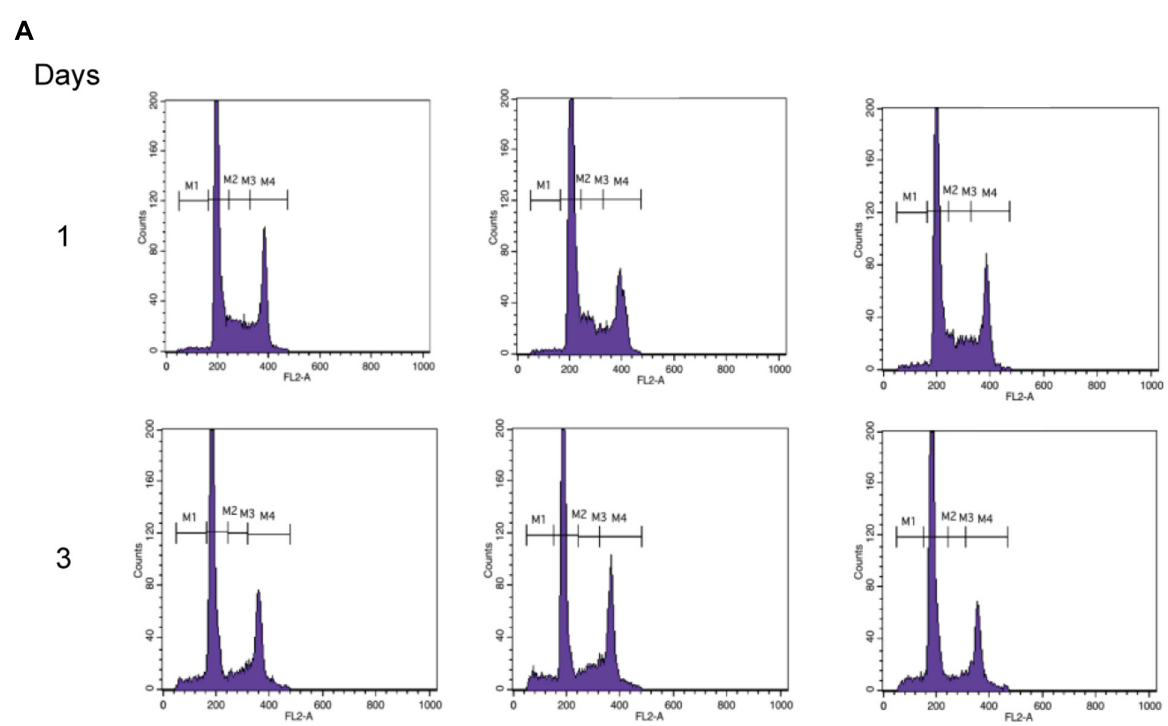

$\operatorname{PTX}(\mathrm{nM})$

0

0.1

0.3

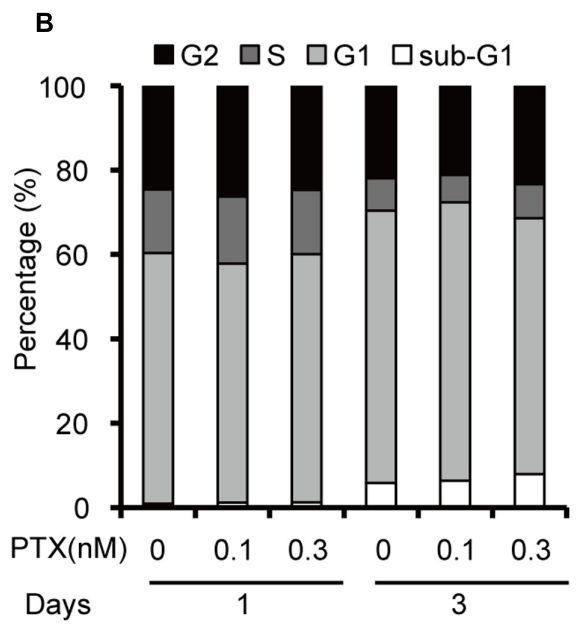

C

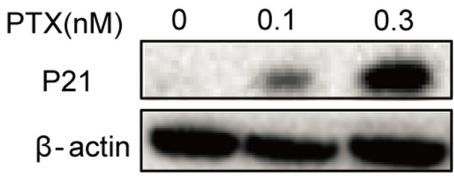

D

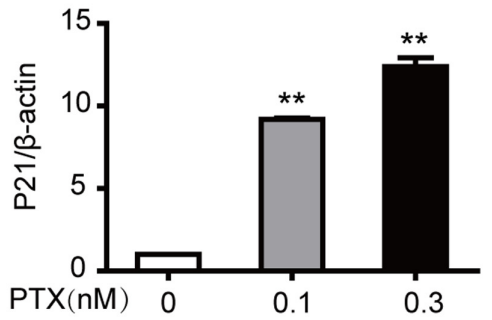

FIGURE 2 | Effects of low-dose PTX on the HCT116 cell cycle. (A) HCT116 cells were exposed to PTX at 0.1 and $0.3 \mathrm{nM}$ for 1 or 3 days and then analyzed with flow cytometric FCM. (M1: sub- $\left.G_{1} ; M 2: G_{0} / G_{1} ; M 3: S ; M 4: G_{2} / M\right)$. (B) The histogram of the cell cycle distribution of HCT116 cells treated with PTX at the indicated concentrations and time. (C) The total protein expression level of p21 in HCT116 cells. HCT116 cells were treated with PTX at the indicated concentrations for 3 days. Whole cell extracts were prepared, and equal amounts of protein were separated on SDS-PAGE and examined by Western blot. The control for protein loading with $\beta$-actin is shown. (D) Quantification of p21 total protein expression level. Data represent the means $\pm \mathrm{SD}, n=3$ independent experiments. ${ }^{*} p<0.05$ and ${ }^{* *} p<0.01$ versus control.

\section{Flow Cytometric Analysis of the Cell Cycle}

The cells were plated into 6-well plates and incubated for 1 and 3 days with nutrient solution containing various concentrations of PTX $(0,0.1$, and $0.3 \mathrm{nM})$. Briefly, the cells were collected and fixed in $0^{\circ} \mathrm{C} 70 \%$ ethanol and stored at $-20^{\circ} \mathrm{C}$. The cells were then washed and resuspended in cold PBS and incubated at $37^{\circ} \mathrm{C}$ for $30 \mathrm{~min}$ with $10 \mathrm{mg} / \mathrm{mL}$ RNase and $1 \mathrm{mg} / \mathrm{mL}$ propidium iodide (Sigma-Aldrich, USA). DNA content analysis was performed by flow cytometry (BD, San Diego, CA, USA). The percentage of cells in different cell cycle phases was determined with Cell Quest acquisition software (BD Biosciences Pharmingen, USA).

\section{Lactate Assay and pH Determination}

Lactate generation was analyzed, following the manufacturer's instructions. In brief, HCT116 cells were treated with low-dose PTX (0.1 and $0.3 \mathrm{nM})$ for $0,1,2$, or 3 days. Then, the culture media supernatant was collected for the detection of lactate generation using a Lactate Assay Kit purchased from Sigma-Aldrich, Inc. (MAK064, St. Louis, MO, USA) and an EnVision Multilabel Reader. Lactate generated was calculated as follows: Lactate generation $(\mathrm{ng} / \mu \mathrm{L})=$ (amount of lactic acid in unknown sample from standard curve/sample volume added into the wells) * lactate molecular weight. 


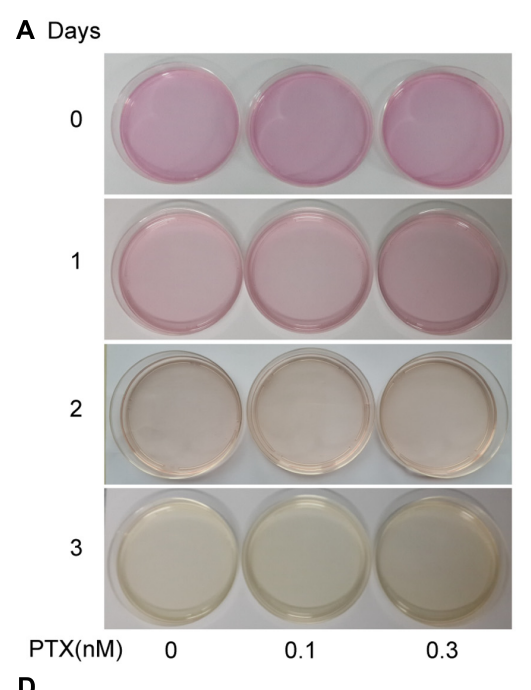

B

D
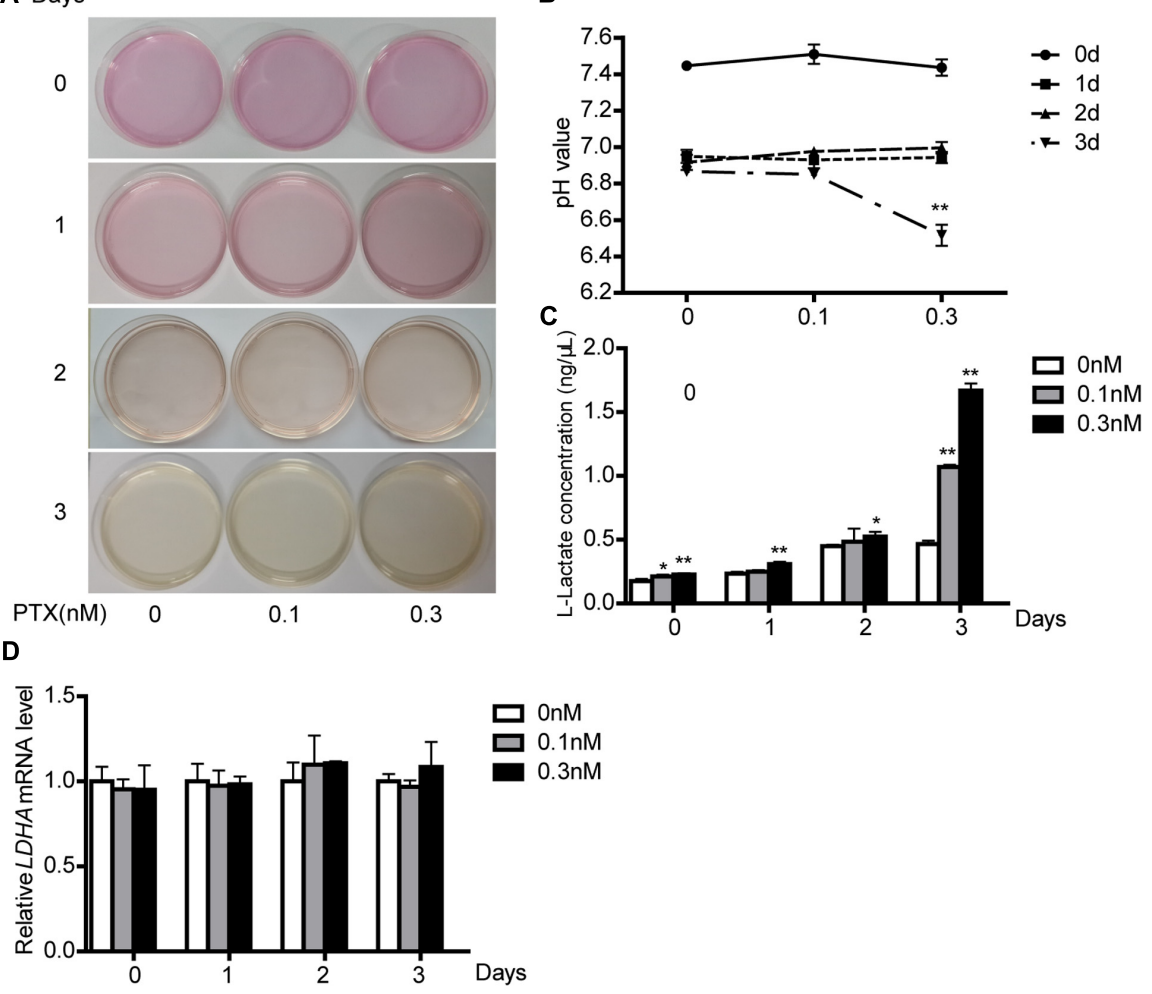

FIGURE 3 | Effect of low-dose PTX on lactate production and LDH activity. (A) HCT116 cells were seeded in $10 \mathrm{~mm}^{2}$ dishes and treated with 0.1 and $0.3 \mathrm{nM}$ PTX for 0,1 , 2, or 3 days. HCT116 cells cultured in medium only served as the control. (B) The medium pH. HCT116 cells treated with low-dose PTX at the indicated concentrations and time. (C) Media were collected and subjected to a lactate assay. (D) After measuring lactate, the cells were harvested, and the mRNA expression level of LDHA was determined by q-PCR. Data represent the means $\pm \mathrm{SD}, n=3$ independent experiments. ${ }^{*} p<0.05$ and ${ }^{* *} p<0.01$ versus control.

Culture media samples treated with PTX were collected at the indicated time points as described above to directly measure their $\mathrm{pH}$ with a meter from Sartorius (PB-10, Göttingen, Germany).

\section{RNA Isolation and Quantitative Real-Time PCR}

HCT116 cells were treated with 0.1 and 0.3 nM PTX for $0,1,2$, or 3 days. Total RNA was isolated using TRIpure reagent (BioTek, China), according to the manufacturer's instructions. The cDNA was synthesized from total RNA using a PrimeScript RT reagent Kit with g Eraser (TaKaRa, Japan). The obtained cDNA was used as a template in SYBR green-based quantitative-polymerase chain reaction (q-PCR) (CFX-96, Bio-Rad, USA). The mRNA expression levels of glycolysis-related genes and glutaminolysisrelated genes were assessed by q-PCR. $\beta$-actin was used for normalization. Primers are listed in Table $\mathbf{1 .}$

\section{Protein Extraction and Immunoblotting}

After HCT116 cells were treated with PTX at the indicated times and concentrations, cells were washed twice with PBS and collected. Then, total protein concentrations of cell lysates were determined with a BCA Protein Assay kit (Beyotime, Shanghai, China). Protein samples (total protein loading of $100 \mu \mathrm{g}$ ) were separated by $10 \%$ SDS-PAGE and transferred onto PVDF membranes. These membranes were incubated for $30 \mathrm{~min}$ in 5\% BSA buffer (Solarbio, Beijing, China) with gentle shaking to block non-specific binding before incubation with the diluted primary antibody (p53: 1:200, p21: 1:200, $\beta$-actin: 1:5000) overnight at $4^{\circ} \mathrm{C}$. Subsequently, membranes were incubated with 5000 -fold diluted secondary antibody (Santa Cruz, CA, USA) for $90 \mathrm{~min}$ at room temperature. The membrane was washed three times in PBS, for 10 min each time. Then, the membrane was treated for 3 min in the dark with reagent from an Easysee Western Blot Kit (Transgene, Alsace, France).

\section{Statistical Analysis}

Statistical comparisons were performed using Student's $t$-test. Quantitative data were expressed as the means \pm SD. $p<0.05$ was considered significant. ${ }^{*} p<0.05$ and ${ }^{* *} p<0.01$ versus controls.

\section{RESULTS}

\section{Effect of Low-Dose PTX on the Morphology and Viability of HCT116 Cells}

Our previous studies demonstrated that 1 and $3 \mathrm{nM}$ PTX had an impact on morphology and viability of colorectal 

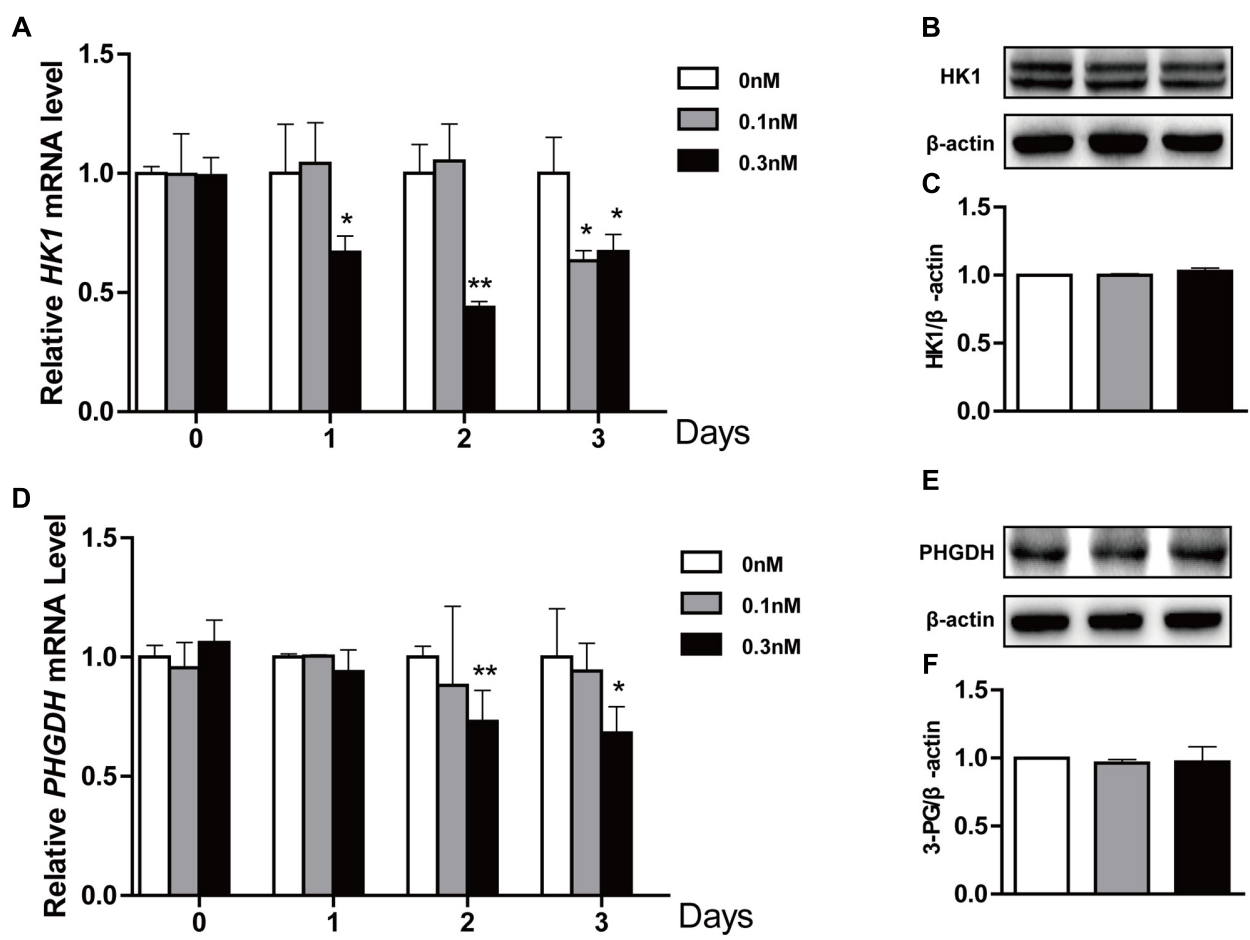

E

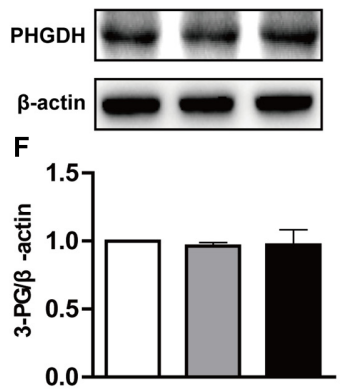

FIGURE 4 | Effect of low-dose PTX on the expression level of glycolysis-related genes in HCT116 cells. HCT116 cells were exposed to PTX at 0.1 and $0.3 \mathrm{nM}$ for $0,1,2$, or 3 days, followed by gene expression levels detected by quantitative real-time PCR assay and Western blot. (A,D) The mRNA expression of HK1 and PHGDH genes. (B,E) The protein expression of HK1 and PHGDH in cells treated with 0.1 and $0.3 \mathrm{nM}$ PTX for 3 days. (C,F) Quantification of the protein expression of $\mathrm{HK} 1$ and PHGDH. Data represent the means $\pm \mathrm{SD}, n=3$ independent experiments. ${ }^{*} p<0.05$ and ${ }^{* *} p<0.01$ versus control.

carcinoma HCT116 cells ( $\mathrm{Li}$ et al., unpublished). Here, we further investigated whether lower doses of PTX had similar effects. Here we found that the low-dose PTX did not alter morphology of HCT116 cells (Figure 1A), however, it significantly decreased colony-forming ability of these cells (Figures 1B,C). Furthermore, the MTT assay further indicated that low-dosage PTX significantly inhibited viability of HCT116 cells in a dose-independent manner (Figure 1D). These findings indicate that low-dose PTX exerts a significant influence on the proliferation of HCT116 cells.

\section{Effect of Low-Dose PTX on the Cell Cycle in HCT116 Cells}

Our previous results indicated that 1 and 3 nM PTX blocked the cell cycle at the $\mathrm{G}_{0} / \mathrm{G}_{1}$ phase, which inhibited colorectal carcinoma cell proliferation (Li et al., unpublished). To investigate effects of low-dose PTX on the cell cycle in HCT116 cells, we also performed an analysis of the cell cycle using flow cytometry (Figure 2A). We found that the sub- $\mathrm{G}_{1}$, $G_{1}, S$ and $G_{2}$ phases of HCT-116 cells were not significantly changed by treatment at indicated concentrations and times (Figure 2B).

Interestingly, these PTX treatments increased levels of p21 protein by 5.87 - and 7.34-fold, in a dose-dependent manner (Figures 2C,D). As an inhibitor of cyclin D/cdk complexes, p21 can influence the cell cycle (Orlando et al., 2015), but our results indicated that the cycle of treated cells did not change. Based on the above findings, we surmised that low-dose PTX acts through another mechanism, other than blocking the cell cycle, to inhibit colorectal carcinoma cell growth.

\section{Effect of Low-Dose PTX on Lactate Production and the mRNA Expression of LDHA}

When HCT116 cells were treated as described Figure $\mathbf{3 A}$, we found that the culture media gradually turned yellow through time. This media color indicated higher acidity. We thus collected the culture media as described above and determined its $\mathrm{pH}$ value. Culture media $\mathrm{pH}$ decreased through time (Figure 3B).

Tumor cell proliferation is susceptible to alterations in microenvironment $\mathrm{pH}$ (Bolzoni et al., 2016). To explore causes of reduced $\mathrm{pH}$, we measured its lactate concentration, because lactate is a major source of acidity in cell metabolism (Seheult et al., 2017). We found that low-dose PTX increased lactate production with dose and time (Figure 3C), consistent with the lowered $\mathrm{pH}$ of the medium. In glucose metabolism, lactate production is primarily affected by the lactate dehydrogenase enzyme (LDH). However, the mRNA expression level of LDHA was not different in cells treated with or without PTX (Figure 3D), which suggests that PTX induces lactate production through a mechanism other than increased expression of $L D H A$. 
A

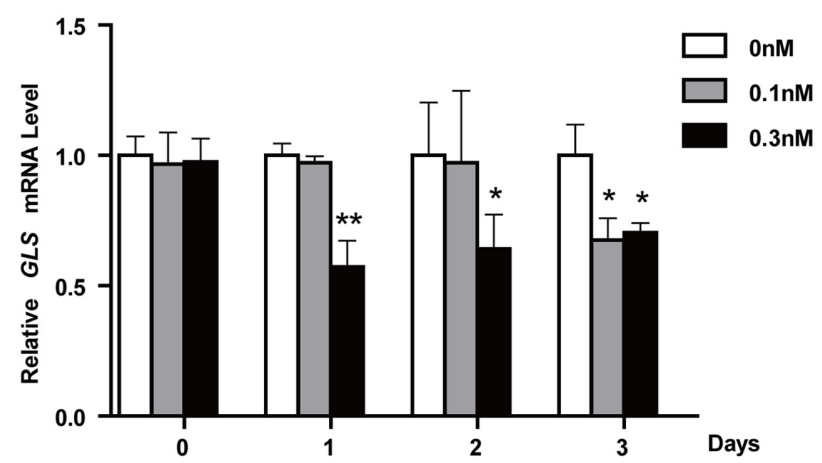

D

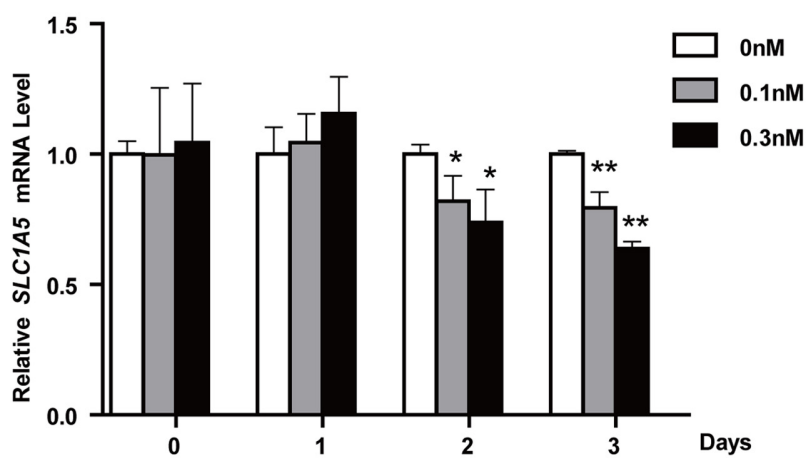

G

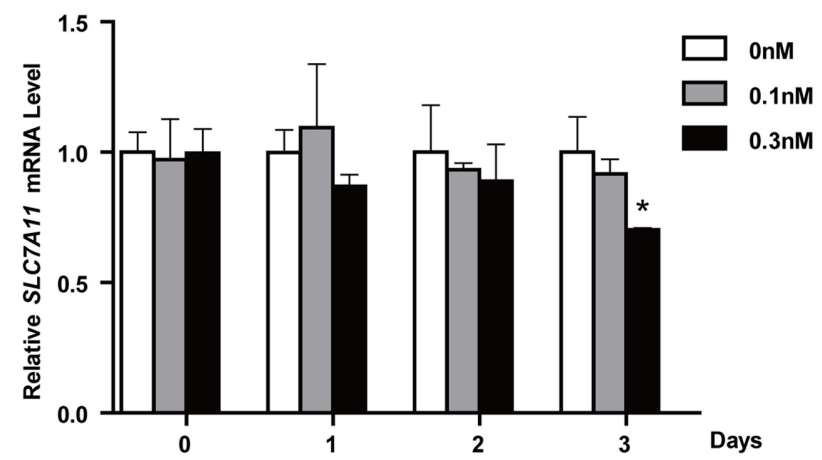

$\mathbf{J}$

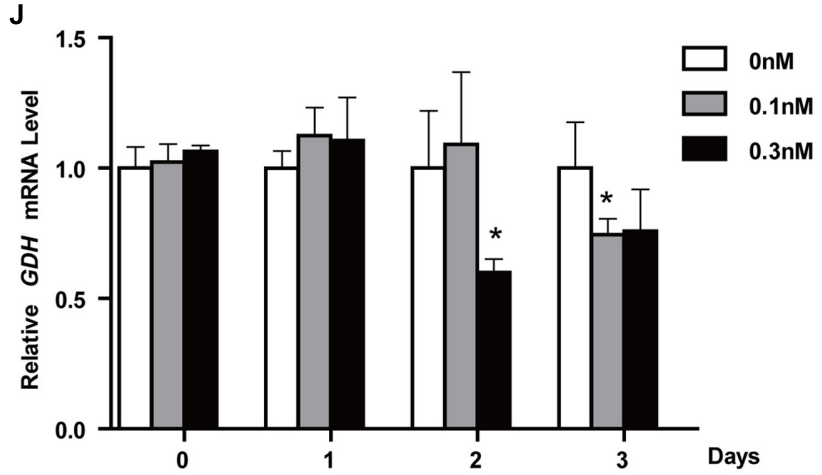

B

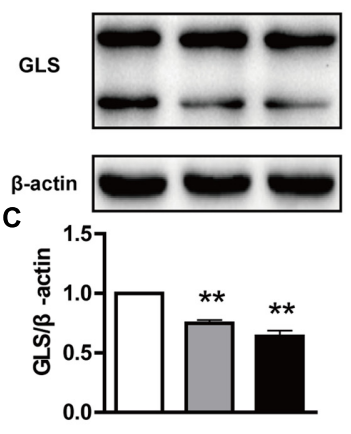

$\mathbf{E}$

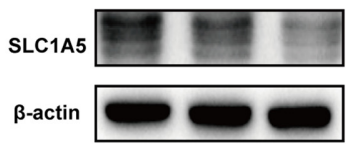

$\mathbf{F}$

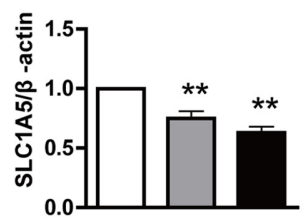

H

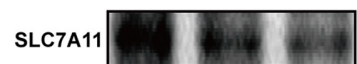

B-actin

I

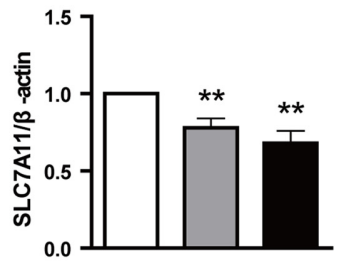

K

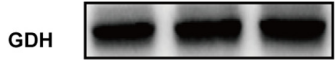

$\beta$-actin

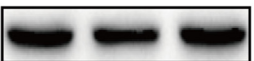

$\mathbf{L}$

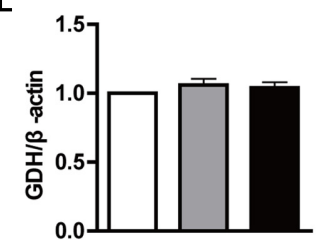

FIGURE 5 | Effects of low-dose PTX on mRNA expression of glutaminolysis-related genes in HCT116 cells. HCT116 cells were treated with 0.1 and $0.3 \mathrm{nM}$ PTX for $0,1,2$, or 3 days, and then mRNA and protein expression of glutaminolysis-related genes was detected by quantitative real-time PCR assay and Western blot. (A,D,G,J) The mRNA expression of GLS, SLC1A5, SLC7A11 and GLUD1 genes. (B,E,H,K) The protein expression of GLS, SLC1A5, SLC7A11 and GLUD1 in cells treated with 0.1 and $0.3 \mathrm{nM}$ PTX for 3 days. (C,F,I,L) Quantification of the protein expression, GLS, SLC1A5, SLC7A11 and GLUD1. Data represent the means $\pm \mathrm{SD}, n=3$ independent experiments. ${ }^{*} p<0.05$ and ${ }^{* *} p<0.01$ versus control. 


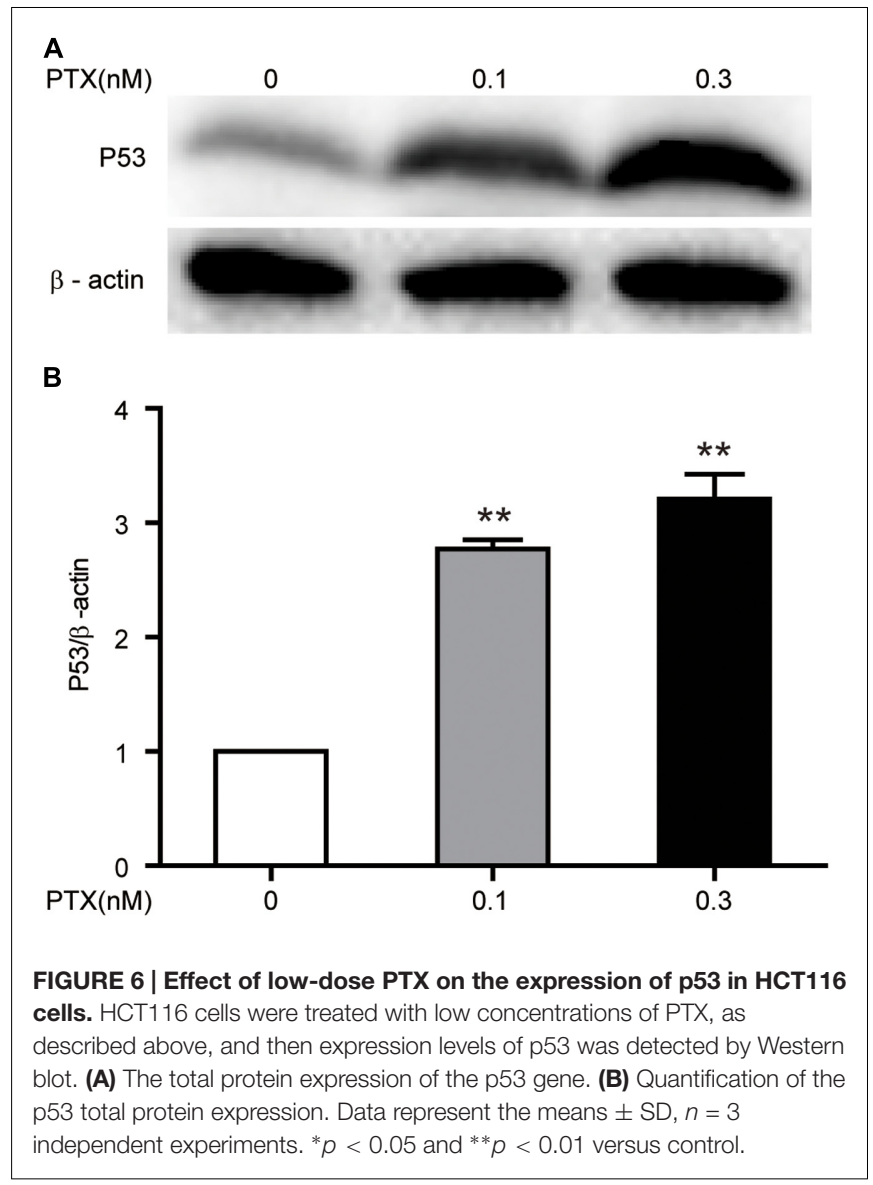

Effect of Low-Dose PTX on Glycolysis-Related Genes in HCT116 Cells

Lactate is final product of glycolysis, and this pathway is regulated by $H K 1, P H G D H, G L U T 1$ and PDK1 among others (Soga, 2013; Wu et al., 2014). Thus, to determine whether lactate production was related to glycolysis, we measured expression levels of glycolysis-related genes in treated cells using qPCR and Western blot. We found that the low-dose PTX significantly decreased mRNA expression of glycolysisrelated genes $H K 1$ and $P H G D H$ in time-dependent manners (Figures 4A,D), however, the protein expression of these genes did not change (Figures $4 \mathbf{B}, \mathbf{C}, \mathbf{E}, \mathbf{F}$ ). Moreover, the mRNA expression of glycolysis-related genes PDK1 and GLUT1 did not change (Supplementary Figure S1). Therefore, pathways other than glycolysis appear to be involved in the PTX-induced lactate production.

\section{Effect of Low-Dose PTX on Glutaminolysis-Related Genes in HCT116 Cells}

To maintain a robust increase in nutrient uptake, in addition to increasing glycolysis, tumor cells commonly activate glutaminolysis. GLS, GDH, SLC7A11 and SLC1A5 genes play an

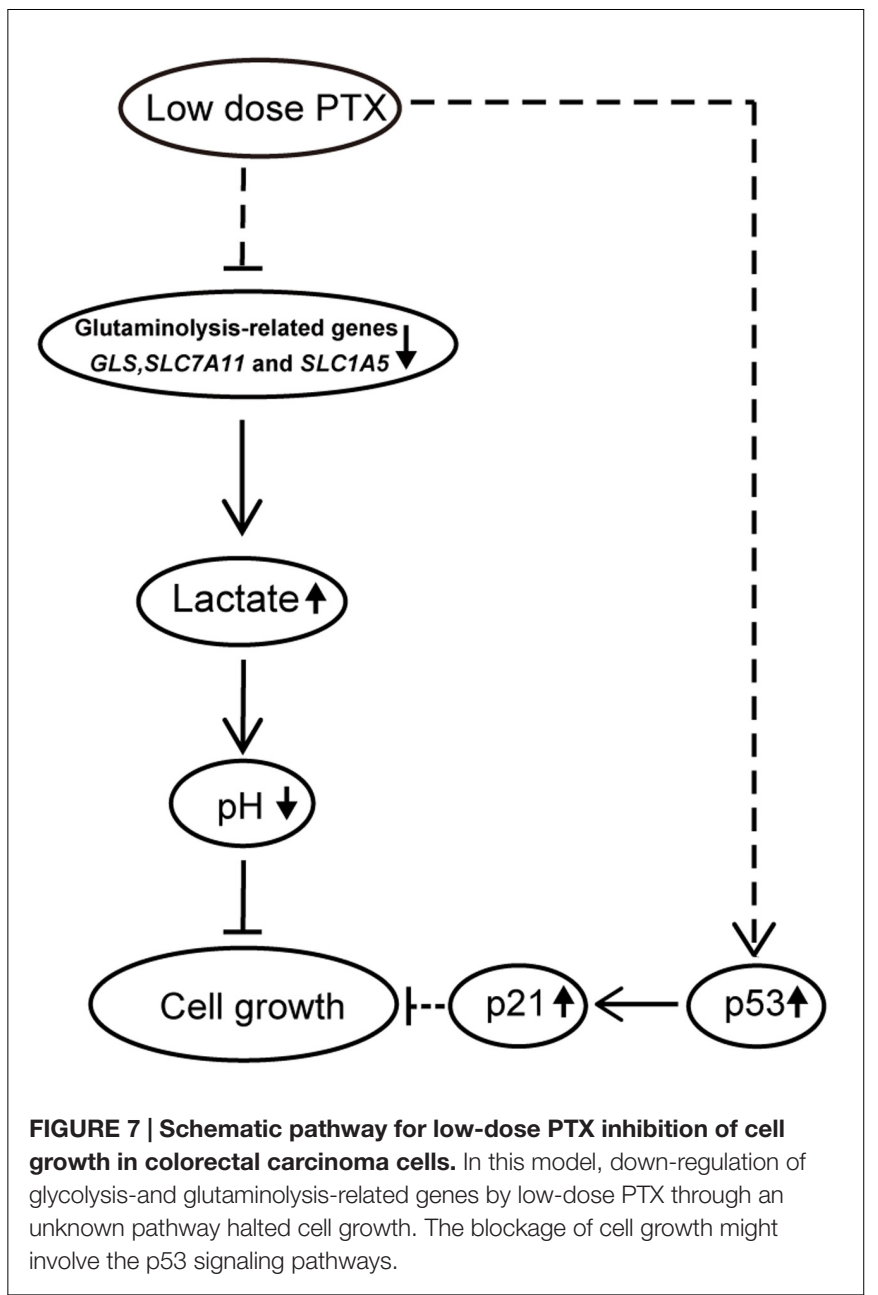

important role in glutamate transport and metabolism (Locasale, 2013). To further explore whether glutaminolysis was related to lactate production, we also treated HCT116 cells as described above and found that low-dose PTX significantly decreased the mRNA of glutaminolysis-related genes GLS and SLC1A5 in a time-dependent manner, SLC7A11 for 3 days at $0.3 \mathrm{nM}$ PTX (Figures 5A,D,G). And the protein expression level of GLS, SLC1A5 and SLC7A11 genes significantly decreased in dose-dependent manner (Figures 5B,C,E,F,H,I). In addition, the mRNA expression of GDH significantly decreased in a time-dependent manner, whereas the protein expression had no change (Figures 5J-L). Our results showed that the low-dose PTX significantly decreased the expression level of glutaminolysis-related genes, promoting lactate production.

\section{Effect of Low-Dose PTX on the Expression of p53 in HCT116 Cells}

Published reports show that PTX inhibits tumor cell growth through up-regulation of tumor-suppressive p53 (Choi and Yoo, 2012). In addition, based on the above finding of an increase in the protein expression level of $\mathrm{p} 21$, which is a downstream gene of $\mathrm{p} 53$, we further verified that low-dose PTX had an impact on 
cell growth through regulation of p53 in PTX-treated HCT116 cells. We detected the protein expression level of p53 in treated cells by Western blot and found that low-dose PTX significantly increased expression level of p53, by 2.34 - to 2.88 -fold, in a dose-dependent manner (Figures 6A,B).

\section{DISCUSSION}

In general, PTX could inhibit proliferation of tumor cell through blocking cell cycle G0/G1 or G2/M phases (Othman et al., 2001). However, we found that low-dose PTX $(0.3 \mathrm{nM})$ inhibited the proliferation of HCT116 cells without cell-cycle change by upregulating expression of p21 proteins (Figures 1, 2A). This may be due to $\mathrm{p} 21$ inhibiting cellular growth by pathways not regulating cell cycle, for instance, during notch 1 activation, p21 suppressed E2F1-dependent Wnt4 expression to inhibit tumor cell proliferation (Ren et al., 2014). p21 also binds to STAT3 inhibiting STAT3-dependent gene expression and preventing cell growth (Siveen et al., 2014). In addition, we also found that the expression of p53 as the upstream gene of p21 was significantly up-regulated by PTX dosage (Figures 6A,B). Therefore, we suggest that low-dose PTX inhibits tumor cell proliferation by significantly increasing $\mathrm{p} 21$ protein expression by up-regulation of $\mathrm{p} 53$. Detailed mechanisms will require further study.

Interestingly, we found that low-dose PTX was able to significantly increase lactate production and decrease media $\mathrm{pH}$ (Figures 3A,B). It has been reported that increased lactate secretion can reduce $\mathrm{pH}$ and thus affect enzymes and coenzymes function, with negative effects on cell metabolism providing energy and materials for tumor cells (Evelyn et al., 2006). Moreover, the lower $\mathrm{pH}$ would affect membrane charge distributions, altering absorption of nutrients and the discharge of metabolites (Sanhueza et al., 2016). The $\mathrm{pH}$ value is an important parameter that significantly influences cell growth, recombinant protein production, cell metabolism and protein glycosylation (Trummer et al., 2006). Our results demonstrate that low-dose PTX affect the growth of HCT116 cells through lowered $\mathrm{pH}$ value.

Recently, it has been reported that many anticancer drugs could inhibit tumor cell growth through inhibition of glutaminolysis (Gill et al., 2016). Moreover, inhibition of glutaminolysis increased lactate production in several types of tumors (Draoui and Feron, 2011; Todaka et al., 2017). Consistent with these results, we found that low-dose PTX down-regulated expression of glutaminolysis-related genes (GLS, SLC7A11 and

\section{REFERENCES}

Abdifard, E., Amini, S., Bab, S., Masroor, N., Khachian, A., and Heidari, M. (2016). Incidence trends of colorectal cancer in Iran during 2000-2009: a populationbased study. Med. J. Islam. Repub. Iran 30, 382.

Altman, B. J., Stine, Z. E., and Dang, C. V. (2016). From Krebs to clinic: glutamine metabolism to cancer therapy. Nat. Rev. Cancer 16, 619-634. doi: 10.1038/nrc. 2016.71

Banerji, A., Lax, T., Guyer, A., Hurwitz, S., Camargo, C. A Jr., and Long, A. A. (2014). Management of hypersensitivity reactions to Carboplatin and Paclitaxel
SLC1A5) and increased lactate production in HCT116 cells (Figures 3C, 5). These findings suggest that the low-dose PTX could inhibit HCT116 cells by targeting glutaminolysis, however, mechanisms remain to be elucidated.

Figure 7 is a schematic representation of proposed molecular basis for the low-dose PTX inhibition of tumor cell growth by regulating glutaminolysis in HCT116 cells. We found that low-dose PTX down-regulated glutaminolysis-related genes (GLS, SLC7A11 and SLC1A5) and increased lactate production, resulting in decreased $\mathrm{pH}$ of the tumor microenvironment which inhibited tumor cell growth. Additionally, up-regulation of p53 and p21 in colorectal carcinoma cells also contributed to this inhibition. We thus present a theoretical basis for further study of anticancer mechanisms of low-dose PTX. In addition, it will facilitate the discovery of "smart" therapeutics that can inhibit tumor energetics and viability.

\section{AUTHOR CONTRIBUTIONS}

$\mathrm{H}-\mathrm{YZ}$ and H-JW conceived and designed the experiments. LC, HQ, WZ, AX, BJ, YQ, and HL performed the experiments. H-YZ, $\mathrm{H}-\mathrm{JW}$, and $\mathrm{KX}$ analyzed the data. $\mathrm{H}-\mathrm{YZ}$ and $\mathrm{WZ}$ wrote the paper. All authors reviewed the manuscript.

\section{ACKNOWLEDGMENTS}

This work was supported by the National Natural Science Foundation of China (81160282 and 31560636), Major Program on Basic Research Projects of Yunnan Province (Grant No. 2014FC006), and Natural Science Foundation Key Project of Yunnan Province (Grant No. 2013FA016).

\section{SUPPLEMENTARY MATERIAL}

The Supplementary Material for this article can be found online at: http://journal.frontiersin.org/article/10.3389/fphar. 2017.00244/full\#supplementary-material

FIGURE S1 | Effect of low-dose PTX on the expression level of glycolysis-related genes PDK1 and GLUT1 in HCT116 cells. HCT116 cells were exposed to PTX at 0.1 and $0.3 \mathrm{nM}$ for $0,1,2$, or 3 days, and then gene expression levels were detected by a quantitative real-time PCR assay. (A) The mRNA expression of HK1 gene. (B) The mRNA expression of GLUT1 gene. Data represent the means $\pm \mathrm{SD}, n=3$ independent experiments. ${ }^{*} p<0.05$ and ${ }^{* *} p<0.01$ versus control.

in an outpatient oncology infusion center: a 5-year review. J. Allergy Clin. Immunol. Pract. 2, 428-433. doi: 10.1016/j.jaip.2014.04.010

Barbuti, A. M., and Chen, Z. S. (2015). Paclitaxel through the ages of anticancer therapy: exploring its role in chemoresistance and radiation therapy. Cancers (Basel) 7, 2360-2371. doi: 10.3390/cancers7040897

Bocci, G., Di Paolo, A., and Danesi, R. (2013). The pharmacological bases of the antiangiogenic activity of paclitaxel. Angiogenesis 16, 481-492. doi: 10.1007/ s10456-013-9334-0

Bolzoni, M., Chiu, M., Accardi, F., Vescovini, R., Airoldi, I., Storti, P., et al. (2016). Dependence on glutamine uptake and glutamine addiction characterize 
myeloma cells: a new attractive target. Blood 128, 667-679. doi: 10.1182/blood2016-01-690743

Chen, X. S., Li, L. Y., Guan, Y. D., Yang, J. M., and Cheng, Y. (2016). Anticancer strategies based on the metabolic profile of tumor cells: therapeutic targeting of the Warburg effect. Acta Pharmacol. Sin. 37, 1013-1019. doi: 10.1038/aps. 2016.47

Choi, Y. H., and Yoo, Y. H. (2012). Taxol-induced growth arrest and apoptosis is associated with the upregulation of the Cdk inhibitor, p21WAF1/CIP1, in human breast cancer cells. Oncol. Rep. 28, 2163-2169. doi: 10.3892/or.2012. 2060

Draoui, N., and Feron, O. (2011). Lactate shuttles at a glance: from physiological paradigms to anti-cancer treatments. Dis. Model Mech. 4, 727-732. doi: 10. $1242 / \mathrm{dmm} .007724$

Duggett, N. A., Griffiths, L. A., Mckenna, O. E., De Santis, V., Yongsanguanchai, N., Mokori, E. B., et al. (2016). Oxidative stress in the development, maintenance and resolution of paclitaxel-induced painful neuropathy. Neuroscience 333, 13-26. doi: 10.1016/j.neuroscience.2016.06.050

Evelyn, T., Katharina, F., Silke, S., Kornelia, S., Christine, L., Renate, K., et al. (2006). Process parameter shifting: part I. effect of DOT, $\mathrm{pH}$ and temperature on the performance of Epo-Fc expressing $\mathrm{CHO}$ cells cultivated in controlled batch bioreactors. Biotechnol. Bioeng. 94, 1033-1044.

Gentric, G., Mieulet, V., and Mechta-Grigoriou, F. (2016). Heterogeneity in cancer metabolism: new concepts in an old field. Antioxid. Redox Signal. 26, 462-485. doi: 10.1089/ars.2016.6750

Gill, K. S., Fernandes, P., O'donovan, T. R., Mckenna, S. L., Doddakula, K. K., Power, D. G., et al. (2016). Glycolysis inhibition as a cancer treatment and its role in an anti-tumour immune response. Biochim. Biophys. Acta 1866, 87-105. doi: 10.1016/j.bbcan.2016.06.005

Gong, L. H., Chen, X. X., Wang, H., Jiang, Q. W., Pan, S. S., Qiu, J. G., et al. (2014). Piperlongumine induces apoptosis and synergizes with cisplatin or paclitaxel in human ovarian cancer cells. Oxid. Med. Cell. Longev. 2014:906804. doi: 10.1155/2014/906804

Jagadish, N., Parashar, D., Gupta, N., Agarwal, S., Suri, V., Kumar, R., et al. (2016). Heat shock protein 70-2 (HSP70-2) is a novel therapeutic target for colorectal cancer and is associated with tumor growth. BMC Cancer 16:561. doi: 10.1186/s12885-016-2592-7

Jiang, H., Tao, W., Zhang, M., Pan, S., Kanwar, J. R., and Sun, X. (2010). Low-dose metronomic paclitaxel chemotherapy suppresses breast tumors and metastases in mice. Cancer Invest. 28, 74-84. doi: 10.3109/07357900902744510

Kalivas, P. W. (2009). The glutamate homeostasis hypothesis of addiction. Nat. Rev. Neurosci. 10, 561-572. doi: 10.1038/nrn2515

Koo, J. S., and Yoon, J. S. (2015). Expression of metabolism-related proteins in lacrimal gland adenoid cystic carcinoma. Am. J. Clin. Pathol. 143, 584-592. doi: 10.1309/AJCPXAYH10WENLTC

Kung, H. N., Marks, J. R., and Chi, J. T. (2011). Glutamine synthetase is a genetic determinant of cell type-specific glutamine independence in breast epithelia. PLoS Genet. 7:e1002229. doi: 10.1371/journal.pgen.1002229

Lien, K., Georgsdottir, S., Sivanathan, L., Chan, K., and Emmenegger, U. (2013). Low-dose metronomic chemotherapy: a systematic literature analysis. Eur. J. Cancer 49, 3387-3395. doi: 10.1016/j.ejca.2013.06.038

Locasale, J. W. (2013). Serine, glycine and one-carbon units: cancer metabolism in full circle. Nat. Rev. Cancer 13, 572-583. doi: 10.1038/nrc3557

Loven, D., Hasnis, E., Bertolini, F., and Shaked, Y. (2013). Low-dose metronomic chemotherapy: from past experience to new paradigms in the treatment of cancer. Drug Discov. Today 18, 193-201. doi: 10.1016/j.drudis.2012. 07.015

Orlando, S., Gallastegui, E., Besson, A., Abril, G., Aligue, R., Pujol, M. J., et al. (2015). p27Kip1 and p21Cip1 collaborate in the regulation of transcription by recruiting cyclin-Cdk complexes on the promoters of target genes. Nucleic Acids Res. 43, 6860-6873. doi: 10.1093/nar/gkv593
Othman, T., Goto, S., Lee, J. B., Taimura, A., Matsumoto, T., and Kosaka, M. (2001). Hyperthermic enhancement of the apoptotic and antiproliferative activities of paclitaxel. Pharmacology 62, 208-212.

Polet, F., Martherus, R., Corbet, C., Pinto, A., and Feron, O. (2016). Inhibition of glucose metabolism prevents glycosylation of the glutamine transporter ASCT2 and promotes compensatory LAT1 upregulation in leukemia cells. Oncotarget 7, 46371-46383. doi: 10.18632/oncotarget.10131

Ren, Z., Kang, W., Wang, L., Sun, B., Ma, J., Zheng, C., et al. (2014). E2F1 renders prostate cancer cell resistant to ICAM-1 mediated antitumor immunity by NF-kappaB modulation. Mol. Cancer 13:84. doi: 10.1186/1476-4598-13-84

Sanhueza, C., Araos, J., Naranjo, L., Barros, E., Subiabre, M., Toledo, F., et al. (2016). Nitric oxide and pH modulation in gynaecological cancer. J. Cell Mol. Med. 20, 2223-2230. doi: 10.1111/jcmm.12921

Seheult, J., Fitzpatrick, G., and Boran, G. (2017). Lactic acidosis: an update. Clin. Chem. Lab. Med. 55, 322-333. doi: 10.1515/cclm-2016-0438

Shen, Y. A., Li, W. H., Chen, P. H., He, C. L., Chang, Y. H., and Chuang, C. M. (2015). Intraperitoneal delivery of a novel liposome-encapsulated paclitaxel redirects metabolic reprogramming and effectively inhibits cancer stem cells in Taxol((R))-resistant ovarian cancer. Am. J. Transl. Res. 7, 841-855.

Siveen, K. S., Sikka, S., Surana, R., Dai, X., Zhang, J., Kumar, A. P., et al. (2014). Targeting the STAT3 signaling pathway in cancer: role of synthetic and natural inhibitors. Biochim. Biophys. Acta 1845, 136-154. doi: 10.1016/j.bbcan.2013. 12.005

Soga, T. (2013). Cancer metabolism: key players in metabolic reprogramming. Cancer Sci. 104, 275-281. doi: 10.1111/cas.12085

Stewart, D. A., Winnike, J. H., Mcritchie, S. L., Clark, R. F., Pathmasiri, W. W., and Sumner, S. J. (2016). Metabolomics analysis of hormone-responsive and triple-negative breast cancer cell responses to paclitaxel identify key metabolic differences. J. Proteome Res. 15, 3225-3240. doi: 10.1021/acs.jproteome. 6 b00430

Todaka, A., Umehara, R., Sasaki, K., Serizawa, M., Urakami, K., Kusuhara, M., et al. (2017). Metabolic profiling of gemcitabine- and paclitaxel-treated immortalized human pancreatic cell lines with K-RASG12D. Biomed. Res. 38, 29-40. doi: 10.2220/biomedres.38.29

Trummer, E., Fauland, K., Seidinger, S., Schriebl, K., Lattenmayer, C., Kunert, R., et al. (2006). Process parameter shifting: part I. effect of DOT, pH, and temperature on the performance of Epo-Fc expressing $\mathrm{CHO}$ cells cultivated in controlled batch bioreactors. Biotechnol. Bioeng. 94, 1033-1044.

Wu, L. E., Gomes, A. P., and Sinclair, D. A. (2014). Geroncogenesis: metabolic changes during aging as a driver of tumorigenesis. Cancer Cell 25, 12-19. doi: 10.1016/j.ccr.2013.12.005

Zhang, Z., Wang, A., Li, H., Zhi, H., and Lu, F. (2016). STAT3-dependent TXNDC17 expression mediates Taxol resistance through inducing autophagy in human colorectal cancer cells. Gene 584, 75-82. doi: 10.1016/j.gene.2016. 03.012

Zhou, J., Chang, L., Guan, Y., Yang, L., Xia, X., Cui, L., et al. (2016). Application of circulating tumor DNA as a non-invasive tool for monitoring the progression of colorectal cancer. PLoS ONE 11:e0159708. doi: 10.1371/journal.pone.0159708

Conflict of Interest Statement: The authors declare that the research was conducted in the absence of any commercial or financial relationships that could be construed as a potential conflict of interest.

Copyright (c) $2017 \mathrm{Lv}, \mathrm{Qu}, \mathrm{Zhu}, \mathrm{Xu}, \mathrm{Xu}$, Jia, Qing, Li, Wei and Zhao. This is an open-access article distributed under the terms of the Creative Commons Attribution License (CC BY). The use, distribution or reproduction in other forums is permitted, provided the original author(s) or licensor are credited and that the original publication in this journal is cited, in accordance with accepted academic practice. No use, distribution or reproduction is permitted which does not comply with these terms. 